\title{
Accuracy of immunological criteria for identifying virological failure in children on antiretroviral therapy - The leDEA Southern Africa Collaboration
}

\author{
Mary-Ann Davies ${ }^{1,{ }^{*}}$, Andrew Boulle ${ }^{1}$, Brian Eley ${ }^{2}$, Harry Moultrie ${ }^{3}$, Karl Technau ${ }^{4}$, Helena \\ Rabie $^{5}$, Gilles van Cutsem ${ }^{1,6}$, Janet Giddy ${ }^{7}$, Robin Wood ${ }^{8}$, Matthias Egger ${ }^{9}$, and Olivia \\ Keiser $^{9}$ for the International epidemiologic Databases to Evaluate AIDS Southern Africa \\ (leDEA-SA) Collaboration
}

\begin{abstract}
${ }^{1}$ School of Public Health and Family Medicine, University of Cape Town, South Africa ${ }^{2}$ Red Cross Children's Hospital and School of Child and Adolescent Health, University of Cape Town, South Africa ${ }^{3}$ Wits Institute for Sexual Reproductive Health, HIV \& Related Diseases, University of the

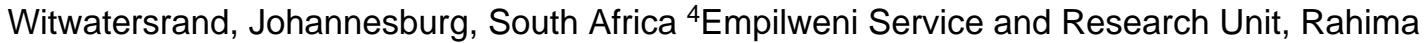
Moosa Mother and Child Hospital, University of the Witwatersrand, Johannesburg, South Africa ${ }^{5}$ Tygerberg Academic Hospital, University of Stellenbosch, Stellenbosch, South Africa ${ }^{6}$ Médecins Sans Frontières, Khayelitsha, South Africa and Khayelitsha ART Programme ${ }^{7}$ McCord Hospital, Durban, South Africa ${ }^{8}$ Gugulethu Community Health Centre and Desmond Tutu HIV Centre, Institute of Infectious Diseases and Molecular Medicine, University of Cape Town, South Africa ${ }^{9}$ Institute of Social and Preventive Medicine, University of Bern, Switzerland
\end{abstract}

\section{Summary}

\begin{abstract}
Objectives-To determine the diagnostic accuracy of World Health Organization (WHO) 2010 and 2006 as well as United States Department of Health and Human Services (DHHS) 2008 definitions of immunological failure for identifying virological failure in children on antiretroviral therapy (ART).
\end{abstract}

\begin{abstract}
Methods-Analysis of data from children ( $<16$ years at ART initiation) at South African ART sites at which CD4 count/percent and HIV-RNA monitoring are performed 6-monthly. Incomplete virological suppression (IVS) was defined as failure to achieve $\geq 1$ HIV-RNA $\leq 400$ copies $/ \mathrm{mL}$ between 6 and 15 months on ART and viral rebound (VR) as confirmed HIV-RNA $\geq 5000$ copies/ $\mathrm{mL}$ in a child on ART for $\geq 18$ months who had achieved suppression during the first year on treatment.
\end{abstract}

\begin{abstract}
Results-Among 3115 children (median (IQR) age 48 (20-84) months at ART initiation) on treatment for $\geq 1$ year, sensitivity of immunological criteria for IVS was $10 \%, 6 \%$ and $26 \%$ for WHO 20062010 and DHHS 2008 criteria respectively. The corresponding positive predictive values (PPV) were 31\% 20\% and 20\%. Diagnostic accuracy for VR was determined in 2513 children with $\geq 18$ months of follow-up and virological suppression during the first year on ART with sensitivity of 5\% (WHO 2006/2010) and 27\% (DHHS 2008). PPV results were 42\% (WHO 2010), 43\% (WHO 2006) and 20\% (DHHS 2008).
\end{abstract}

Conclusion-Current immunological criteria are unable to correctly identify children failing ART virologically. Improved access to viral load testing is needed to reliably identify virological failure in children.

*Corresponding author: University of Cape Town Faculty of Health Sciences, Anzio Road, Observatory, 7925, Cape Town, South Africa. Phone: +27 21 4066051; Fax: +27 21 4066764; mary-ann.davies@uct.ac.za. 


\section{Keywords}

children; antiretroviral therapy; immunological criteria; sensitivity, specificity; virological failure

\section{Introduction}

Poor access to viral load testing in resource-limited settings results in reliance on immunological criteria to identify treatment failure in patients on antiretroviral therapy (ART). Studies in adults have shown limited value of immunological criteria to detect virological failure (VF) (Keiser et al. 2009, Mee et al. 2008, Badri et al. 2008). While low sensitivity of immunological criteria for identifying VF could result in delayed switching to second-line treatment with accumulation of resistance mutations, low positive predictive value (PPV) may incorrectly identify patients as needing second-line treatment when they are virologically suppressed. The few paediatric studies of diagnostic accuracy of immunological criteria for VF are limited by small cohort size and/or VF definition based on single elevated HIV-RNA measurements (Ruel et al. 2010, Jittamala et al. 2009, Emmett et al. 2010). We analysed data from routine pediatric ART clinics to determine the diagnostic accuracy of WHO $(2006,2010)$ and United States Department of Health and Human Services (DHHS) 2008 (National Institutes of Health 2008) criteria for immunological failure for identifying (i) incomplete viral suppression during the first year on ART and (ii) confirmed viral rebound.

\section{Methods}

Data was collected prospectively from ART-naïve children ( $<16$ years at ART start) initiating $\geq 3$ antiretrovirals at South African sites participating in IeDEA-Southern Africa (IeDEA-SA, see www.iedea-sa.org), all of which had CD4 and HIV-RNA measurements performed 6-monthly. The characteristics of these sites have been described previously (Davies et al. 2009). Each site has institutional ethical approval to contribute data to IeDEA analyses.

HIV-RNA was measured using Amplicor 1.5 (Roche Diagnostics) or NucliSens EasyQ assays (bioMerieux), with good comparability (Stevens et al. 2005). CD4 measurements were performed using standard dual platform flow cytometry or the single platform PanLeucogated method (Glencross et al. 2008). We defined incomplete virological suppression (IVS) as failure to achieve $\geq 1$ HIV-RNA $\leq 400$ copies $/ \mathrm{mL}$ between 6 and 15 months on ART and viral rebound (VR) as confirmed HIV-RNA 25000 copies $/ \mathrm{mL}$ in a child on ART for $\geq 18$ months whose HIV-RNA had suppressed during the first year on treatment. We examined the following immunological criteria: WHO 2010: No definition for children $<2$ years; CD4\% $<10 \%$ or CD $4<200$ cells $/ \mathrm{mm}^{3}$ (age 2-4 years); CD4<100 cells/ $\mathrm{mm}^{3}$ (age $\geq 5$ years) (WHO 2010). WHO 2006: No definition for children <1 year; CD4\% $<15 \%$ (age 1-2 years); CD4\%<10\% (age 3-4 years); CD4<100 cells/mm3 (age $\geq 5$ years) (WHO 2006). DHHS: During first year on ART: $<5$ percentage point CD4\% increase from baseline (CD4\% $<15$ and age $<5$ years at baseline) or $<50$ cells $/ \mathrm{mm}^{3} \mathrm{CD} 4$ increase from baseline $\left(\mathrm{CD} 4<200\right.$ cells $/ \mathrm{mm}^{3}$ and age $\geq 5$ years at baseline); decline of CD4\% by 5 percentage points from previous value, confirmed at subsequent measurement $(<365$ days after first low value) (any age); return of CD4 count sbaseline value (age $\geq 5$ years at baseline) (National Institutes of Health 2008).

For IVS, we assessed the diagnostic accuracy of never achieving a CD4 above the immunological thresholds between 6 and 15 months on ART for identifying a child who never had HIV-RNA $\leq 400$ copies/mL during the same period. Only children followed up for 
$\geq 1$ year with $\geq 1$ HIV-RNA measurement between 6 and 15 months were included. For VR, CD4 and HIV-RNA measurements were carried forward for up to 3 months where tests were performed asynchronously. For each unique paired CD4 and HIV-RNA measurement, we determined the diagnostic value of CD4-based criteria for VR, using robust standard errors to account for multiple measures per patient. Immunological results for which there was still no concurrent virological diagnosis after carrying forward results, were not evaluated. Further, the last immunological result before the end of follow-up/data base closure was excluded to ensure that there was sufficient follow-up to for a confirmatory low viral load measurement to have been done. Separate sensitivity analyses were performed requiring either consecutive (within 365 days) CD4 measurements meeting immunological criteria or using an HIV-RNA threshold of 1000 copies/mL to define confirmed VR.

\section{Results}

Of 3640 children with $\geq 1$ year of follow-up, 3115 (86\%) had $\geq 1$ HIV-RNA measurement between 6 and 15 months on ART. At ART initiation, median (interquartile range (IQR)) age was 48 (20-84) months. Most children were severely ill at ART initiation: 81\% had WHO-defined severe immune suppression $(\mathrm{n}=2911)$ and $68 \%$ of children had WHO Clinical Stage $3 / 4$ disease $(n=2294)$ (WHO 2006). In keeping with South African guidelines recommending protease inhibitor- (PI-) based first-line therapy in children $<3$ years old (National Department of Health South Africa 2005), 35\% of children were on PI-based firstline treatment with non-nucleoside reverse transcriptase-based therapy in the remainder. IVS occurred in $12.6 \%$ of children and sensitivity of immunological criteria for identifying IVS ranged from 6\% (WHO 2010) to 26\% (DHHS) and PPV from 20\% (WHO 2010 and DHHS) to $31 \%$ (WHO 2006) (Table 1 (a)).

The accuracy of immunological criteria for identifying VR was assessed in 2513 children with at least $\geq 18$ months follow-up on ART whose HIV-RNA had suppressed during the first year on treatment. The cumulative probability of viral rebound in the following 2 years (by 42 months since ART start) was 5.5\% (95\% CI: 4.2-7.1). Requiring consecutive CD4 counts to meet immunological criteria increased PPV only slightly at the expense of sensitivity, without any improvement in the area under the receiver operating characteristic curve (Table 1 (b)).Sensitivity of immunological criteria for identifying VR ranged from 5\% (WHO 2006/2010) to 27\% (DHHS) and PPV from 20\% (DHHS) to 43\% (WHO 2006) (Table 1(b)). Lowering the VR threshold to 1000 copies/mL and using confirmed CD4 values to define immunological criteria yielded sensitivity $(95 \% \mathrm{CI})$ of $2 \%(0-5 \%)$ (WHO 2006); $2 \%$ (0-4\%) (WHO 2010) and 14\% (8-19\%) (DHHS); and PPV (95\% CI) of 90\% (72-100\%) (WHO 2006); 89\% (68-100\%) (WHO 2010) and 38\% (26-50\%) (DHHS).

\section{Discussion}

In this large longitudinal study we found that sensitivity and PPV were low for identifying VF using WHO and DHHS immunological criteria. For example, using confirmed HIVRNA 25000 copies/mL after initial virological suppression to define VR, sensitivity was only 4\% using either WHO 2006 or 2010 criteria. Fewer than half of children meeting WHO 2006/2010 criteria would have HIV-RNA 25000 copies/mL.

These results concur with those of previous small studies from resource-limited settings. Among 116 children in Uganda $20(17 \%)$ had sustained viraemia ( $\geq 400$ copies $/ \mathrm{mL}$ ) beyond 24 weeks on ART (Ruel et al. 2010). Only 2 of these ever met WHO 2006 immunological criteria, and did so after $>550$ days of viraemia, while none met WHO 2010 criteria (Ruel et al. 2010). Similarly, in a cross-sectional study of 206 children in Tanzania on ART for a median duration of 2.4 years, $32 \%$ had a single HIV-RNA $\geq 400 \mathrm{copies} / \mathrm{mL}$ (Emmett et al. 
2010). WHO 2006 clinical and immunological failure criteria combined had a PPV of $100 \%$ but identified only $3.5 \%$ of children with HIV-RNA $\geq 400$ copies $/ \mathrm{mL}$ (Emmett et al. 2010). In Thailand, the sensitivity and PPV of DHHS immunological criteria for identifying children with a single HIV-RNA $>1000$ copies $/ \mathrm{mL}$ was $15 \%$ and $16 \%$ respectively (Jittamala et al. 2009).

The poor sensitivity of immunological criteria for identifying VF is disappointing, however perhaps an even greater concern is preventing the incorrect diagnosis of treatment failure in a virologically suppressed child, with unnecessary switch to second-line treatment, hence the importance of PPV. While PPV reached $89 \%$ for identifying VR $\geq 1000$ copies $/ \mathrm{mL}$ using a confirmed CD4 value meeting WHO 2010 criteria, given that the WHO 2010 guidelines HIV-RNA threshold for switching to second-line is 5000 copies $/ \mathrm{ml}$, in most instances the number of false positives using immunological criteria is unacceptably high. Further, absence of immunological failure on ART provides no assurance that a child is virologically suppressed and not accumulating resistance mutations, and even high CD4 thresholds (e.g. DHHS criteria) have low sensitivity for VF (PENPACT1 Study Team 2011). Improved access to VL monitoring both to assess adherence and identify VF is therefore needed (Ford \& Calmy 2010, Wilson et al. 2009).

There are several limitations to this analysis of routinely collected data. Missing baseline CD4 values limited evaluation of DHHS criteria. Baseline CD4 values are often unavailable to clinicians if children initiate ART on clinical criteria, records are lost or a child changes treatment site after ART initiation, highlighting the value of simple criteria using current or recent measurements. The accuracy of WHO 2006 and 2010 criteria could not be evaluated in children $<1$ and $<2$ years old respectively. Work-up bias may occur if either the reference (HIV-RNA) or index (CD4) tests are not applied consistently (Whiting et al. 2004). In particular, rigorous confirmation of low CD4 values is seldom done in South Africa, due to access to HIV-RNA measurements to diagnose treatment failure. Exclusion of intercurrent illness as a cause of low CD4, as advised in WHO guidelines (WHO 2010), was not possible due to limited data on episodes of clinical illness. Immunological criteria may have performed better if only low CD4 counts not explained by intercurrent illness had been considered. PPV is dependent on the VF incidence in different programs, however the incidence in this cohort was similar to that of other studies (Davies et al. 2011, Jittamala et al. 2009, Kamya et al. 2007).

In summary, our results suggest that current immunological criteria are unable to correctly identify children failing ART virologically. Improved access to viral load testing appears to be the only feasible approach at this stage for reliably identifying VF in children on ART. There are several existing technologies for the measurement of viral load (Stevens and Marshall 2010) and expanded access should be supported by governments and donors.

\section{Acknowledgments}

This study was supported by the National Institute of Allergy and Infectious Diseases and the Eunice Kennedy Shriver National Institute of Child Health and Human Development (grant 1 U01 AI069924-01). The funders had no role in study design, data collection and analysis, decision to publish, or preparation of the manuscript. We thank all the children whose data was used in this analysis, as well as their caregivers. We also thank all staff at participating sites for providing clinical patient care and preparation of data contributed to the IeDEA Southern Africa collaboration. Many thanks to Nicola Maxwell for preparing the combined data for analysis, and to Morna Cornell and Claire Graber for project management. 


\section{References}

Badri M, Lawn SD, Wood R. Utility of CD4 cell counts for early prediction of virological failure during antiretroviral therapy in a resource-limited setting. BMC.Infect.Dis. 2008; 8:89. 89. [PubMed: 18601727]

Davies M, Keiser O, Technau K, et al. Outcomes of the South African National Antiretroviral Treatment (ART) programme for children - The IeDEA Southern Africa Collaboration. S Afr Med J. 2009; 99:730-737. [PubMed: 20128272]

Davies M, Moultrie H, Eley B, et al. Virologic failure and second-line antiretroviral therapy in children in South Africa: The IeDEA Southern Africa collaboration. J Acquir Immune Defic Syndr. 2011; 56:270-278. [PubMed: 21107266]

Emmett SD, Cunningham C, Mmbaga BT, et al. Predicting virologic failure among HIV-1 infected children receiving antiretroviral therapy in Tanzania: a cross-sectional study. J Acquir Immune Defic Syndr. 2010; 54:368-375. [PubMed: 20216225]

Ford N, Calmy A. Improving first-line antiretroviral therapy in resource-limited settings. Curr Opin HIV AIDS. 2010; 5:38-47. [PubMed: 20046146]

Glencross DK, Janossy G, Coetzee LM, et al. Large-scale affordable PanLeucogated CD4+ testing with proactive internal and external quality assessment: in support of the South African national comprehensive care, treatment and management programme for HIV and AIDS. Cytometry B Clin Cytom. 2008; 74:S131-S140. [PubMed: 18228566]

Jittamala P, Puthanakit T, Chaiinseeard S, Sirisanthana V. Predictors of virologic failure and genotypic resistance mutation patterns in Thai children receiving non0nucleoside reverse transcriptase inhibitor-based antiretroviral therapy. Pediatr Infect Dis J. 2009; 28:826-830. [PubMed: 19654564]

Kamya MR, Mayanja-Kizza H, Kambugu A, et al. Predictors of long-term viral failure among ugandan children and adults treated with antiretroviral therapy. J Acquir Immune Defic Syndr. 2007; 46:187-193. [PubMed: 17693883]

Keiser O, MacPhail P, Boulle A, et al. Accuracy of WHO CD4 cell count criteria for virological failure of antiretroviral therapy. Trop Med Int Health. 2009; 14:1220-5. [PubMed: 19624478]

Mee P, Fielding KL, Charalambous S, Churchyard GJ, Grant AD. Evaluation of the WHO criteria for antiretroviral treatment failure among adults in South Africa. AIDS. 2008; 22:1971-1977. [PubMed: 18784460]

National Department of Health South Africa. Guidelines for the management of HIV-infected children in South Africa. Jacana; 2005.

National Institutes of Health. [Accessed 2008/17/12] Guidelines for the use of antiretroviral agents in Pediatric HIV infection (Online). 2008. NIH. Available: http://AIDSinfo.nih.gov

PENPACT1 Study Team. First-line antiretroviral therapy with a protease inhibitor versus nonnucleoside reverse transcriptase inhibitor and switch at higher versus low viral load in HIVinfected children: an open-label, randomised phase 2/3 trial. Lancet Infect Dis. 2011; 11:273-283. [PubMed: 21288774]

Ruel, TD.; Achan, J.; Charlebois, E.; Havlir, D.; Kamya, M. Sustained viremia is common among HIV-infected Ugandan children receiving antiretroviral therapy and not detected by WHO CD4 criteria. 18th International AIDS Conference; Vienna Austria. 2010.

Stevens W, Wiggill T, Horsfield P, Coetzee L, Scott LE. Evaluation of the NucliSensEasyQ assay in HIV-1-infected individuals in South Africa. Journal of Virological Methods. 2005; 124:105-110. [PubMed: 15664057]

Stevens WS, Marshall TM. Challenges in implementing HIV load testing in South Africa. J Infect Dis. 2010; 201(Suppl 1):S78-84. [PubMed: 20225952]

Whiting P, Rutjes AW, Reitsma JB, Glas AS, Bossuyt PM, Kleijnen J. Sources of variation and bias in studies of diagnostic accuracy: a systematic review. Ann Intern Med. 2004; 140:189-202. [PubMed: 14757617]

WHO. Antiretroviral therapy of HIV infection in infants and children: towards universal access (Online). WHO; Switzerland: 2006. Available: http://www.who.int/hiv/pub/paediatric/infants/en/ index.html [Accessed 2007/09/02] 
WHO. [Accessed 2010/19/10 2010] Antiretroviral therapy for HIV infection in infants and children: Towards universal access. Recommendations for a public health approach: 2010 revision. 2010. (Online). Available: http://www.who.int/hiv/pub/paediatric/infants2010/en/index.html

Wilson D, Keiluhu AK, Kogrum S, et al. HIV-1 viral load monitoring: an opportunity to reinforce treatment adherence in a resource-limited setting in Thailand. Trans R Soc Trop Med Hyg. 2009; 103:601-6. [PubMed: 19110288] 


\section{Table 1(a)}

Diagnostic value of immunological criteria for identifying children with incomplete viral suppression during first year on ART

\begin{tabular}{|c|c|c|c|c|c|c|}
\hline & \multicolumn{3}{|c|}{$\begin{array}{c}\text { Comparison of immunological failure diagnosis } \\
\text { (based on highest CD4 between } 6 \text { and } 15 \text { months on } \\
\text { ART) } \\
\text { with failure of viral suppression in same period } \\
\end{array}$} & \multicolumn{3}{|c|}{$\begin{array}{c}\text { Comparison of immunological failure diagnosis } \\
\text { (based on highest CD4 between } 6 \text { and } 15 \text { months on } \\
\text { ART } \\
\text { and confirmed on a subsequent measurement) } \\
\text { with failure of viral suppression in same period } \\
\end{array}$} \\
\hline & WHO $2006^{\dagger}$ & WHO $2010^{\S}$ & USA & WHO 2006 ${ }^{\dagger}$ & WHO $2010^{\S}$ & USA \\
\hline Number of children (\%) & 90/2714 (3.3) & $61 / 2380(2.6)$ & $355 / 2585$ (13.7) & $13 / 2502(0.5)$ & 9/2217 (0.4) & $72 / 2277(3.2)$ \\
\hline $\begin{array}{l}\text { Number of children with } \\
\text { paired data }\end{array}$ & 2581 & 2256 & 2470 & 2405 & 2126 & 2190 \\
\hline Sensitivity (\%) & $10(7-14)$ & $6(3-10)$ & $26(21-31)$ & $2(0-4)$ & $2(0-4)$ & $9(5-12)$ \\
\hline Specificity (\%) & $97(97-98)$ & $98(97-98)$ & $87(86-89)$ & $99(99-100)$ & $99(99-100)$ & $97(97-98)$ \\
\hline PPV $(\%)$ & $31(22-41)$ & $20(10-30)$ & $20(16-24)$ & $38(8-69)$ & $33(0-72)$ & $27(16-38)$ \\
\hline NPV (\%) & $90(89-91)$ & $92(91-93)$ & $91(89-92)$ & $90(89-91)$ & $92(91-93)$ & $90(89-92)$ \\
\hline Number true positives & 28 & 12 & 69 & 5 & 3 & 19 \\
\hline Number true negatives & 2242 & 2017 & 1928 & 2151 & 1945 & 1916 \\
\hline Number false negatives & 250 & 179 & 197 & 241 & 172 & 204 \\
\hline Number false positives & 61 & 48 & 276 & 8 & 6 & 51 \\
\hline $\mathrm{LR}+$ & 3.8 & 2.7 & 2.07 & 5.49 & 5.57 & 3.29 \\
\hline LR - & 0.92 & 0.95 & 0.85 & 0.98 & 0.99 & 0.94 \\
\hline Area under ROC curve & 0.537 & 0.520 & 0.567 & 0.508 & 0.507 & 0.530 \\
\hline \multicolumn{7}{|c|}{$\begin{array}{l}\text { Number of children with paired data (both CD4 and HIV-RNA measures available and CD4 criteria evaluable in terms of immunological criteria) } \\
\text { liffers for different definitions because of different age and data requirements for each definition }\end{array}$} \\
\hline \multicolumn{7}{|c|}{ Only determined for children $>12$ months of age } \\
\hline \multicolumn{7}{|c|}{$\xi_{\text {Only determined for children }>24 \text { months of age }}$} \\
\hline
\end{tabular}




\section{Table 1(b)}

Diagnostic value of immunological criteria for identifying children with viral rebound

\begin{tabular}{|c|c|c|c|c|c|c|}
\hline & \multicolumn{3}{|c|}{$\begin{array}{l}\text { Comparison of immunological failure diagnosis } \\
\text { (based on single CD4 measure meeting criteria) } \\
\text { with confirmed HIV-RNA >5,000 copies/ml } \\
\end{array}$} & \multicolumn{3}{|c|}{$\begin{array}{l}\text { Comparison of immunological failure diagnosis } \\
\text { (based on consecutive CD4 measure meeting criteria } \\
\text { with confirmed HIV-RNA >5,000 copies } / \mathrm{ml} \\
\end{array}$} \\
\hline & WHO 2006 & WHO $2010^{\S}$ & USA & WHO 2006 & WHO $2010^{\S}$ & USA \\
\hline $\begin{array}{l}\text { Cumulative probability by } \\
2 \text { years }\end{array}$ & $2.2 \%(1.5-3.2)$ & $2.3 \%(1.5-3.3)$ & $\begin{array}{c}28.1 \%(25.2- \\
31.3)\end{array}$ & $0.4 \%(0.2-1.0)$ & $0.3 \%(0.1-0.9)$ & $10.4 \%(8.6-12.6)$ \\
\hline $\begin{array}{l}\text { Number of evaluable } \\
\text { pairs of data* }\end{array}$ & 2499 & 2593 & 2191 & 2395 & 2507 & 2110 \\
\hline Sensitivity (\%) & $5(2-9)$ & $5(2-9)$ & $27(19-35)$ & $2(0-5)$ & $2(0-4)$ & $13(6-21)$ \\
\hline Specificity (\%) & $99(99-100)$ & $99(99-100)$ & $88(86-90)$ & $100(99-100)$ & $100(99-100)$ & $95(94-96)$ \\
\hline $\operatorname{PPV}(\%)$ & $43(23-63)$ & $42(22-61)$ & $20(13-26)$ & $50(19-81)$ & $44(14-75)$ & $21(10-32)$ \\
\hline NPV $(\%)$ & $91(89-93)$ & $91(89-93)$ & $92(90-94)$ & $91(89-93)$ & $91(89-93)$ & $91(89-93)$ \\
\hline Number true positives & 13 & 13 & 58 & 5 & 4 & 26 \\
\hline Number true negatives & 2245 & 2327 & 1740 & 2168 & 2269 & 1816 \\
\hline Number false negatives & 224 & 235 & 157 & 217 & 229 & 172 \\
\hline Number false positives & 17 & 18 & 236 & 5 & 5 & 96 \\
\hline $\mathrm{LR}+$ & 7.3 & 6.83 & 2.26 & 9.79 & 7.81 & 2.62 \\
\hline LR - & 0.95 & 0.95 & 0.83 & 0.98 & 0.98 & 0.91 \\
\hline Area under ROC curve & 0.524 & 0.522 & 0.575 & 0.51 & 0.508 & 0.541 \\
\hline
\end{tabular}

